\title{
Determinants of Dietary Adherence Among Type 2 Diabetes Patients Aimed COVID-19 at the University of Gondar Comprehensive Specialized Hospital
}

This article was published in the following Dove Press journal:

Diabetes, Metabolic Syndrome and Obesity: Targets and Therapy

\section{Chilot Kassa Mekonnen (iD) Yohannes Mulu Ferede Hailemichael Kindie Abate \\ Department of Medical, Nursing, School of Nursing, College of Medicine and Health Sciences, University of Gondar, Gondar, Ethiopia}

Background: People with diabetes are proving to be at higher risk of severe coronavirus infection than the general population. Non-adherence to dietary recommendation is a critical problem in individuals with type 2 diabetes; particularly, in the era of coronavirus. This study aimed to explore determinants that affect adherence to the recommendations of diet during coronavirus pandemic.

Objective: This study aimed to determine dietary adherence among diabetes type 2 patients aimed at coronavirus pandemic and its associated factors.

Methods: An institutional-based cross-sectional study was conducted among type 2 diabetes patients on follow-up. Systematic random sampling was employed to get representative study participants. Dietary adherence was dichotomized and with logistic regression analysis to assess the association between dependent and explanatory variables. The degree of association was interpreted by using both crude odds ratios and adjusted odds ratios with $95 \%$ confidence intervals and P-values $<0.05$ were considered statistically significant.

Results: In this study, 576 participants took part with a $99.3 \%$ response rate. The dietary adherence was found to be $48.3 \%$ with [95\% CI (44.1-52.4)]. The educational level of College and above was $[\mathrm{AOR}=3.64,95 \% \mathrm{CI}(1.59-8.34)]$, being a government-employed [AOR=2.38, 95\% CI (1.13-4.99)], being urban [AOR=1.30, 95\% CI (1.09-2.42)], family size of less than five $[\mathrm{AOR}=1.27,95 \%$ CI (1.08-1.97)], medium level of wealth-status $[\mathrm{AOR}=2.26,95 \% \mathrm{CI}(1.67-4.54)]$, family history of diabetes [AOR=2.18, 95\% CI (1.14-$4.19)$ ], duration of diabetes more than 15 years $[\mathrm{AOR}=5.39,95 \% \mathrm{CI}(1.57-18.52)]$, and knowledge related to coronavirus $[\mathrm{AOR}=4.33,95 \% \mathrm{CI}(2.71-6.92)]$ were significantly associated with adherence to the dietary recommendation.

Conclusion: The dietary adherence was relatively low aimed at coronavirus pandemic. Knowledge about COVID-19 was found significantly associated with dietary adherence.

Recommendations: There has to be a strong commitment to aware the community to minimize the risk of coronavirus severity among diabetes patients. This could be through adherence to dietary recommendations of particularly in the era of coronavirus pandemic.

Keywords: adherence, diet, COVID-19, coronavirus, diabetes, type 2, Ethiopia

\section{Introduction}

People with diabetes are proved to be at a higher risk of severe coronavirus (COVID19) infection than the general population. ${ }^{1}$ Non-adherence to the dietary recommendation is a critical problem for individuals with type 2 diabetes; particularly, in the era 
of the COVID-19. ${ }^{2}$ This study aimed to explore determinants that affect adherence to the recommendation of diet during the COVID-19 pandemic. Although there are uncertainties that COVID-19 has associated with a worse outcome of people with diabetes. The effect of non-adherence on glycemic control also plays a significant role in worse outcomes of COVID-19 disease in diabetes patients. ${ }^{3}$ The COVID-19 pandemic imposed a very critical challenge in the provision of care for patients with diabetes mellitus. ${ }^{4,5}$ Moreover, those with uncontrolled diabetes are at a higher risk of complications related to COVID-19 disease. ${ }^{3,6,7}$ The rapid spreading of the COVID-19 worldwide has brought many consequences on the care of chronic diseases like diabetes. $^{2,6}$ A piece of evidence showed that diabetes has ranked second most common comorbidity among COVID19 patients to impose severity after cardio-metabolic diseases. ${ }^{8}$ Diabetes is a chronic disease that occurs either when the pancreas does not produce enough insulin or cannot use it properly leading to hyperglycemia. ${ }^{9}$ Around 422 million people worldwide have diabetes; in America, almost 16 million peoples have Type 2 diabetes; ${ }^{10}$ according to the International Diabetes Federation (IDF) data, 19 million adults (20-79) are living with diabetes in the Africa Region. ${ }^{11}$ In Ethiopia, the prevalence of diabetes ranged from $2.0 \%$ to $6.5 \%$ with a low $2 \%$ in smaller rural areas. ${ }^{12}$ This rise in prevalence is due to aging populations and lifestyle changes, including unhealthy diets and lack of awareness about dietary recommendation. Overweight and obesity are the strongest risk factors for Type 2 diabetes mellitus; it has been estimated at $60-90 \%$ risk variation. ${ }^{13}$ Adherence to a healthy diet and regular physical exercise are ways to prevent or delay the onset of type 2 diabetes. ${ }^{9,14}$ A diet rich in vegetables (these should take up half of the daily plate!), fruit, lean protein, whole grains, low-fat dairy in moderate amounts, and healthy fats from things like avocado and nuts is best. ${ }^{15,16}$ Additionally, getting 30 minutes of regular exercise per day (think brisk walking, strength training, stretching exercise, etc.) at least 5 days a week can keep blood glucose level normal, and lower the risk of developing diabetes by $35 \% .{ }^{16,17}$ Evidence showed that $50 \%$ of patients with type 2 diabetes were not made changes to their diet. ${ }^{18}$ Despite this fact, in European regions, the rates of non-adherence to diet recommendations were ranging from $24 \%$ to $88 \% .^{17,19-22}$ Similarly, in African regions, nearly $37 \%$ of type 2 diabetic patients did not adhere to dietary recommendations. ${ }^{23-25}$ Likewise, in Ethiopia regions, $74.3 \%$ of diabetes patients poorly adhered to the dietary recommendations. ${ }^{26-28}$ Concerning the prevalence of dietary non-adherence, in Europe and African countries, the prevalence of dietary non-adherence was ranging from $25 \%$ to $84.8 \%$ and $16.19 \%$ to $52 \%$, respectively, in Ethiopia, it ranged from $46.8 \%$ to 64.3\%. ${ }^{17,20,21,23,29}$ Dietary adherence was influenced by the factors like residence, occupation, duration of illness, living distance from the hospital, sex, age, marital status, patients advice by a physician, family/social support, patient perception on the role of diet, level of education, diabetes education, duration of illness, nutritional education, social support, make choices when eating out, co-morbidities, wealth status were factors of dietary nonadherence. ${ }^{17,19-21,23,25,27,29-32}$ To overcome such types of non-adherence problems; health professionals' were struggling by providing continuous health education on lifestyle modification. But still, we do not achieve the desired outcome. ${ }^{33-35}$

Now a time in the era of the COVID-19 patients' healthy lifestyle could be compromised, for instance, patients may have dietary feeding changes following this disease. Also, there were no studies which were investigated the prevalence and factors of dietary non-adherence among type 2 diabetes patients with the ear of COVID- 19 . High emphases have been given to social distancing, personal hygiene, facemask utilization, and quarantine, but not about dietary recommendations. ${ }^{36}$ Moreover, it is the researchers' responsibility to where we are in recommending dietary adherence, particularly in the area of a COVID19 pandemic. On that, the government, policymakers, and health professionals will work to solve the problems early before the COVID-19 caused more morbidity and mortality among type 2 diabetic patients. Therefore, the current study was conducted to assess the prevalence and predictors of adherence to Dietary recommendations among Type 2 Diabetes adult Patients at the University of Gondar Comprehensive Specialized Hospital, Northwest Ethiopia.

\section{Methods \\ Study Setting}

The study was conducted at the University of Gondar Comprehensive Specialized Hospital. This is the most visited, Hospital in Amhara regional state. The hospital serves more than seven million people in and outside of the catchment area. It provides promotive, preventive, curative and rehabilitative services to the catchment population. The target population was adult type 2 diabetes 
patients who attended a follow-up clinic in the former mentioned Hospital during the data collection period. Permonth there were around (1200) type 2 diabetes patients observed in the diabetic clinic at the Hospital.

\section{Study Design and Period}

An institution-based cross-sectional study was conducted from August 20/2020 to September 20/2020.

\section{Source Population}

All diabetes patients, who had follow-up at the Hospital, were considered as the source population.

\section{Study Population}

Randomly selected type 2 diabetes patients who attend the follow-up clinic during the data collection period were included in the study.

\section{Inclusion and Exclusion Criteria}

All type 2 diabetes patients who had at least one follow-up visit after diagnosis were included in the study, whereas those newly diagnosed patients on the date of data collection were excluded from the study. Those patients with gestational diabetes were also excluded from the study.

\section{Sample Size Determination and Sampling Technique}

The required sample size of the study was determined by using a single population proportion formula with the assumptions of a $95 \%$ of confidence interval, $4 \%$ margin of error(d), and prevalence of dietary adherence (P) was taken as $64.3 \%$ from the study conducted in Jimma ${ }^{32}$ and adding 5\% non-response rate. By using these all the final sample size was 582. Per-month there were around 1500 type 2 diabetes patients visited the follow-up clinic of the hospital (68 diabetes patients per day $* 22$ working days) observed in the diabetic clinic.

Then, taking the total diabetes patients who visited the diabetes clinic the K-value was calculated as total diabetes patients who visited per-month (1500)/sample size $(582)=3$ and then using the lottery method from 1 to 5 the starting point was fixed to be 3 . Then, each study participant was selected every 3rd person pattern using a systematic random sampling technique based on the order of coming into the diabetic follow-up clinic. To avoid recycling of data due to double interviewing special markers were used in the chart of interviewed diabetes patients. It was also strengthened by verbal confirmation whether they interviewed in the previous consecutive data collection days or not at all.

\section{Operational Definition}

Adherence: The behavior that the patient did what has been recommended by the health professionals to do so.

Diet: The recommended diet for people with diabetes in which they ate small meals spread through the day (at least 5/days), ate fruits and vegetables daily and ate foods high in fibres and whole grain but low in fats, sugars and carbohydrates that have a high glycemic index.

Dietary adherence: Participants who scored $>15$ from 20 correctly answered items, "partial adherence" if the participants scored between 10 and 15 correctly answered items. Dietary non-adherence: The participants scored $<10$ from 20 correctly answered items. ${ }^{17}$

COVID-19-related knowledge: The overall knowledge level was assessed using the mean score of knowledge question due to the symmetrical nature of the data $(\geq 12.25 \pm 3.45)$ was good knowledge and $<12.25 \pm 3.45$ was poor knowledge). ${ }^{37}$

Wealth status: It was computed using the principal component analysis from the variables of monthly income, agricultural productivity, household assets, and utility by checking the necessary assumptions of (PCA) and the percentile group of sum up was classified as low wealth status based on the first percent quartile as, 2nd percentile quartile medium based on the 2 nd percentile group and the 3rd percent quartile as high-level wealth status. ${ }^{38}$

The habit of drinking alcohol: Among the participants those who drunk $\geq 3$ drinks per day(female), $\geq 4$ drinks per day for male considered as alcohol drunker in this study. ${ }^{39}$

Physical exercise: Those participants who did physical exercise five days per week considered they had engaged in physical exercise. ${ }^{22}$

\section{Data Collection Tools and Procedures}

The questionnaire was adapted from related and previously published studies. ${ }^{17,32,40}$ It included six sections (Supplementary File1); the first sections include the sociodemographic-related information of the study participants. The second section included items that assessed the dietary adherence to the recommendations during the months preceding the study. The third section encompasses the items that assessed coronavirus (COVID-19)-related knowledge. The fourth section was about respondents' health-related information, the fifth section was behavioral and food-related information, and the six sections were socio-economic status/wealth 
status measuring tool. Dietary adherence was rated at three points Likert scale, which thought to determine whether the patients (always $=0$, sometimes $=1$ or never $=2$ ) followed the recommended dietary advice. The overall score ranged from " 0 to 20 ", the participants' adherence was classified as "good adherence" if they scored $>15$ from correctly answered items, "partial adherence" if the participants scored between 10 and 15 correctly answered items and "non-adherence" if participants scored $<10$ correctly answered items. It had 20 items with the Cronbach's Alpha of (0.876). The third section encompasses the items that assessed COVID-19-related knowledge. The questionnaire assesses 16 knowledge questions with answered "Yes" (1 point) and "No" (0 points) option. The total knowledge scores range from 0 to 16 . The overall knowledge level was assessed using the mean score of knowledge question due to the symmetrical nature of the data $(\geq 12.25 \pm 3.45$ was good knowledge and $(<12.25 \pm 3.457)$ was poor knowledge).

\section{Data Quality Control}

To ensure the quality of data, a pre-test was conducted using $5 \%$ (21) of the sample of diabetes patients outside of the main study participants, before two weeks to assess instrument simplicity, flow and consistency and questionnaire modification was made after the pre-test accordingly. Four data collectors (diploma nurses) and two supervisors (BSc. Nurses) were selected and two days of training about the objectives of the study, how to approach the study participants and to exercise the interview was given to them. The completeness of the questionnaire was checked by supervisors and principal investigators every day and an incomplete questionnaire was discarded.

\section{Data Management and Analysis}

The collected quantitative data were coded and entered into the computer using EPI data version 7. Then, it was exported into the windows of Statistical Package for Social Science (SPSS) version 20 for data analysis. The frequency, percentage, mean and standard deviation were described using tables, figures, and graphs. The model fitness was checked by using Hosmer-Lemeshow goodness of fit test with $(\mathrm{P}=0.76)$ and interpreted as the model was well fitted. To run logistic regression analysis the chi-square assumption was checked. The multicollinearity assumptions were also checked by using variance inflation factor (VIF) and the value was in the range 1-10, interpreted as there was no collinearity. Then, logistic regression analysis was applied to assess the association between dependent and explanatory variables. The degree of association was interpreted by using both crude odds ratios and adjusted odds ratios with 95\% confidence intervals and P-values $<0.05$ were considered statistically significant. Both Bivariate and multivariate analyses were applied to determine factors affecting recommended dietary adherence.

\section{Result \\ Socio-Demographic Characteristics}

Among 576 study participants, 344 (59.7\%) and 246 $(42.7 \%)$ were female and cannot read and write, respectively. The mean age in years was $(51.63 \pm 10.81)$ with a range of $30-77$. More than half $(79.5 \%)$ of the participants were urban dwellers. The vast majority (79.2\%) was in the age category of $>40$ years old (Table 1 ).

\section{Dietary Adherence of Participants}

In this study, the overall dietary adherence was found to be $48.3 \%$ with [95\% CI (44.1-52.4)]. Participants of $25.7 \%$ and $31.1 \%$ were forgetting their meal plan always and sometimes, respectively. Below half, $43.2 \%$ of the participants never forget their meal plan during the era of a COVID-19 pandemic by far. In the participants, $30.4 \%$, and $43.1 \%$ had missed to include fruits in their meals, but 26.6\% never missed (Table 2). Among the study participants, $51.7 \%$ did not adhere to dietary recommendations. The rest $32.6 \%$ were partially adhering and $15.6 \%$ had good adherence to dietary recommendations. Among the study participants, 42.9\% were responding that the food to be cut down a lot has to be sweets, but $13.9 \%$ did not know which food has to be cut down a lot (Supplementary File 2: Figure S1).

\section{Health Status and Available Health Information}

More than half $(52.3 \%)$ of the participants had less than five years of diabetes follow-up, whereas $71.9 \%$ had gotten diabetic health education of health professionals. Only $31.4 \%$ and $28.3 \%$ had a diabetes complication and a family history of diabetes (Table 3 ).

\section{Participants Behavioral and Food-Related Information}

Among the study participants, $2.3 \%$ and $10.6 \%$ had a habit of smoking cigarette and drinking alcohol, respectively. The vast majority, $70.5 \%$ of the participants did a physical exercise to control their blood glucose level. 
Table I Socio-Demographic Characteristics of Participants at the University of Gondar Comprehensive Specialized Hospital, Gondar, Ethiopia, $2020(n=576)$

\begin{tabular}{|c|c|c|}
\hline Variables & Frequency & Percentages (\%) \\
\hline \multicolumn{3}{|l|}{ Sex } \\
\hline Male & 232 & 40.3 \\
\hline Female & 344 & 59.7 \\
\hline \multicolumn{3}{|l|}{ Age in years } \\
\hline$\leq 40$ & 120 & 20.8 \\
\hline$>40$ & 456 & 79.2 \\
\hline \multicolumn{3}{|l|}{ Educational status } \\
\hline Cannot read \& write & 246 & 42.7 \\
\hline Primary school & 35 & 6.1 \\
\hline Secondary school & 44 & 7.6 \\
\hline College \&above & 251 & 43.6 \\
\hline \multicolumn{3}{|l|}{ Marital status } \\
\hline Single & 40 & 6.9 \\
\hline Married & 447 & 77.6 \\
\hline Divorced & 32 & 5.6 \\
\hline Widowed & 57 & 9.9 \\
\hline \multicolumn{3}{|l|}{ Religious } \\
\hline Orthodox & 391 & 67.9 \\
\hline Muslim & 146 & 25.3 \\
\hline Protestant & 39 & 6.8 \\
\hline \multicolumn{3}{|l|}{ Residence } \\
\hline Urbane & 458 & 79.5 \\
\hline Rural & 118 & 20.5 \\
\hline \multicolumn{3}{|l|}{ Occupation } \\
\hline Government employee & 224 & 38.9 \\
\hline Unemployed & 352 & 61.1 \\
\hline \multicolumn{3}{|l|}{ Family size } \\
\hline$<5$ & 297 & 51.6 \\
\hline$\geq 5$ & 279 & 48.4 \\
\hline \multicolumn{3}{|l|}{ Wealth-status } \\
\hline Low & 177 & 30.7 \\
\hline Medium & 198 & 34.4 \\
\hline High & 201 & 34.9 \\
\hline \multicolumn{3}{|l|}{ Ethnic group } \\
\hline Amhara & 500 & 86.8 \\
\hline Oromo & 31 & 5.4 \\
\hline Tigray & 23 & 4.0 \\
\hline Others & 22 & 3.8 \\
\hline
\end{tabular}

Of the study participants, only $18.9 \%$ had their glucometer to check the blood glucose level at home. The majority, $74.6 \%$ of the participants were not experiencing the difficulty of choosing foods appropriate for controlling blood glucose level during the era of COVID-19 (Table 4).
Table 2 Participants' Response of Dietary Adherence Aimed at COVID-19 Pandemic $(n=576)$

\begin{tabular}{|c|c|c|c|}
\hline \multirow{3}{*}{$\begin{array}{l}\text { Aimed COVID-I9 } \\
\text { Did You }\end{array}$} & \multicolumn{3}{|l|}{ Response } \\
\hline & Always & Sometimes & Never \\
\hline & 0 & I & 2 \\
\hline $\begin{array}{l}\text { Forget to plan the meals } \\
\text { you eat ahead. }\end{array}$ & $148(25.7 \%$ & $179(31.1 \%)$ & $249(43.2 \%)$ \\
\hline $\begin{array}{l}\text { Miss your dietary plan } \\
\text { yesterday. }\end{array}$ & $158(27.4 \%)$ & $221(38.4 \%)$ & $197(34.2 \%)$ \\
\hline $\begin{array}{l}\text { Over the past two } \\
\text { weeks, where there } \\
\text { were days you did not } \\
\text { take your dietary plan } \\
\text { properly. }\end{array}$ & $157(27.3 \%)$ & $290(50.3 \%)$ & $129(22.4 \%)$ \\
\hline $\begin{array}{l}\text { Sometimes forget to } \\
\text { comply with your } \\
\text { dietary plan with } \\
\text { everyday life. }\end{array}$ & $203(35.2 \%)$ & $248(43.1 \%)$ & $125(21.7 \%)$ \\
\hline $\begin{array}{l}\text { Feel like your DM is } \\
\text { under control, do you } \\
\text { sometimes stop taking } \\
\text { your dietary plan. }\end{array}$ & $238(41.3 \%)$ & $191(33.2 \%)$ & I47(25.5\%) \\
\hline $\begin{array}{l}\text { Ever feel hassled about } \\
\text { sticking to your diet } \\
\text { plan. }\end{array}$ & $93(16.1 \%)$ & $290(50.3 \%)$ & $193(33.5 \%)$ \\
\hline $\begin{array}{l}\text { Feelings of dietary } \\
\text { restriction. }\end{array}$ & $85(14.8 \%)$ & $260(45.1 \%)$ & $23 I(40.1 \%)$ \\
\hline $\begin{array}{l}\text { Miss to include fruits in } \\
\text { your food daily. }\end{array}$ & $175(30.4 \%)$ & $248(43.1 \%)$ & $153(26.6 \%)$ \\
\hline $\begin{array}{l}\text { Miss to include } \\
\text { vegetables in your food } \\
\text { daily. }\end{array}$ & $|8|(3 \mid .4 \%)$ & $227(39.4 \%)$ & $168(29.2 \%)$ \\
\hline $\begin{array}{l}\text { Forget to cut down the } \\
\text { butter and fat intake in } \\
\text { your food. }\end{array}$ & $190(33.0 \%)$ & $202(35.1 \%)$ & $184(31.9 \%)$ \\
\hline
\end{tabular}

Abbreviations: COVID-19, coronavirus; DM, diabetes mellitus.

\section{Factors Associated with the Dietary Adherence Among Diabetes Type 2 Patients in the Era of COVID-19 Pandemic}

In multivariable analysis, being college and above educational level was 3.64 [AOR=3.64, 95\% CI (1.59-8.34)] times more to adhere to dietary recommendations compared to its counterparts. Being a government-employed was found to be 2.38 times $[\mathrm{AOR}=2.38,95 \% \mathrm{CI}(1.13-4.99)]$ more to 
Table 3 Health Status and Available Health Information of Type 2 Diabetes Patients $(\mathrm{N}=576)$

\begin{tabular}{|c|c|c|c|}
\hline \multirow{2}{*}{$\begin{array}{l}\text { Variables } \\
\text { Duration of DM }\end{array}$} & \multicolumn{2}{|l|}{ Frequency } & \multirow{2}{*}{$\begin{array}{l}\text { Percentages } \\
52.3\end{array}$} \\
\hline & $<5$ years & 301 & \\
\hline & $5-10$ years & 202 & 35.1 \\
\hline & $\mathrm{II}-15$ years & 52 & 9.0 \\
\hline & $>15$ years & 21 & 3.6 \\
\hline \multirow{2}{*}{$\begin{array}{l}\text { Presence of other chronic } \\
\text { illness }\end{array}$} & Yes & 125 & 30.9 \\
\hline & No & 279 & 69.1 \\
\hline \multirow[t]{2}{*}{ Presence of DM complication } & Yes & $|8|$ & 31.4 \\
\hline & No & 395 & 68.6 \\
\hline \multirow{2}{*}{$\begin{array}{l}\text { Have you got an education } \\
\text { about DM? }\end{array}$} & Yes & 414 & 71.9 \\
\hline & No & 162 & 28.1 \\
\hline \multirow{2}{*}{$\begin{array}{l}\text { Do you have a family } \mathrm{Hx} \text { of } \\
\text { DM? }\end{array}$} & Yes & 163 & 28.3 \\
\hline & No & 413 & 71.7 \\
\hline \multirow{2}{*}{$\begin{array}{l}\text { Do you have a journal } \\
\text { pamphlet about DM? }\end{array}$} & Yes & 54 & 13.4 \\
\hline & No & 350 & 86.6 \\
\hline \multirow{2}{*}{$\begin{array}{l}\text { Do you get education from } \\
\text { TV about DM? }\end{array}$} & Yes & 153 & 37.9 \\
\hline & No & 251 & 62.1 \\
\hline \multicolumn{4}{|l|}{ Do you have family support? } \\
\hline & Yes & 375 & 65.1 \\
\hline & No & 201 & 34.9 \\
\hline
\end{tabular}

Abbreviations: DM, diabetes mellitus; TV, television.

adhere with dietary recommendation compared to its counterparts. Being an urban residency was $30 \%[\mathrm{AOR}=1.30$, 95\% CI (1.09-2.42)] more to adhere to the dietary recommendation compared to rural. Having a family size of less than five was found to be $27 \%$ [AOR=1.27, 95\% CI (1.08-1.97)] more to adhere to the dietary recommendation compared to more than five family size. Being in the lower medium level of wealth-status was found to be [AOR $=3.67,95 \%$ CI $(1.54-7.45)]$ more to adhere to the dietary recommendation compared with its counterparts, whereas having a medium level of wealth-status was nearly two $[\mathrm{AOR}=2.26,95 \% \mathrm{CI}(1.67-4.54)]$ times to adhere to the dietary recommendation compared to its counterparts. Having a history of diabetes was nearly two $[\mathrm{AOR}=2.18$, 95\% CI (1.14-4.19)] more to adhere to the dietary recommendation compared to no family history. Having no diabetes-related complication was found nearly two
$[\mathrm{AOR}=1.94,95 \% \mathrm{CI}(1.04-3.61)]$ more to adhere to the dietary recommendation compared to its counterpart. Duration of diabetes more than 15 years was found to be nearly five times $[\mathrm{AOR}=5.39,95 \% \mathrm{CI}(1.57-18.52)$ more to adhere to the dietary recommendation compared to its counterparts. Knowledge related to COVID-19 was found to be 4.33 times $[\mathrm{AOR}=4.33,95 \% \mathrm{CI}(2.71-6.92)]$ more to adhere to the dietary recommendation compared to its counterparts (Table 5).

\section{Discussion}

The dietary adherence in this study was $48.3 \%$ with [ $95 \%$ CI (44.1-52.4)] among type 2 diabetes patients during the era of the COVID-19 pandemic. The finding was lower than the study conducted in Southern Ethiopia 64\%, Ghana $65.1 \%$, Surate city $76.2 \%$, and Botswana $63 \%$ of the participants' adhered to the dietary recommendation, respectively. ${ }^{19,21,22,32}$ The possible explanations might be the time gap and the occurrence of COVID-19 which might affect the overall Socio-demographic activities as well as dietary servings. As evidence revealed that the COVID-19 pandemic has already changed the dietary pattern and habit of the people due to look down and decrease the supply of foods recommended to the diabetes patients by far. Therefore, adherence might be affected by this and that reason. ${ }^{41}$ Moreover, the study conducted in Surat city included both diabetes patients as a study population (both Type 1 and 2) which might be the possible variation in the proportion of adherence. There was also sociodemographic and socio-cultural variation between the current study and the studies mentioned above. But, it was higher than the studies conducted in Dire Adwa 37.5\% and Yemen $21.0 \%$ of the participants adhered to a dietary recommendation. ${ }^{17,28}$ The possible explanation for this variation might be because currently, people have become more aware of the severity of COVID-19 among diabetes patients through different information channels.

It was also higher than the study in Debre Tabor $25.7 \%$ of the participants adhered to a dietary recommendation. ${ }^{26}$ This might be due to time variation and measuring tool cut of point variation. It might also be because people are more sensitive and gather information regarding diabetes and COVID-19 relation from media, health professionals, friends, and relatives than previously did. The habit and lifestyle of the people have also changed due to the COVID-19 pandemic lockdown and availability of the food servings unlike the previous. ${ }^{42}$ The study conducted in Italian also revealed that dietary adherence has been 
Table 4 Frequency Distribution of Participants' Behavioral and Food-Related Information Aimed at COVID-I9 Pandemic $(n=576)$

\begin{tabular}{|c|c|c|c|}
\hline Variables & \multicolumn{2}{|l|}{ Frequency } & Percentage (\%) \\
\hline \multirow[t]{2}{*}{ Habit of smoking } & Yes & 13 & 2.3 \\
\hline & No & 563 & 97.7 \\
\hline \multirow[t]{2}{*}{ A habit of drinking alcohol } & Yes & 61 & 10.6 \\
\hline & No & 515 & 89.4 \\
\hline \multirow[t]{2}{*}{ Physical exercise } & Yes & 406 & 70.5 \\
\hline & No & 170 & 29.5 \\
\hline \multirow[t]{2}{*}{ Considered holidays as other free days in celebration } & Yes & 135 & 23.4 \\
\hline & No & 441 & 76.6 \\
\hline \multirow[t]{2}{*}{ Number of days of exercise per week } & $<5$ days per week & 211 & 52.0 \\
\hline & $\geq 5$ days per week & 195 & 48.0 \\
\hline \multirow[t]{2}{*}{ Number of minutes of exercise per day in five days } & $<30$ minutes per day & 43 & 22.1 \\
\hline & $\geq 30$ minutes per day & 152 & 77.9 \\
\hline \multirow[t]{2}{*}{ Checked FBG every day } & Yes & 66 & 11.5 \\
\hline & No & 510 & 88.5 \\
\hline \multirow[t]{2}{*}{ Do you have a glucometer } & Yes & 109 & 18.9 \\
\hline & No & 467 & 81.1 \\
\hline \multirow[t]{3}{*}{ FBS level } & $<126 \mathrm{mg} / \mathrm{del}$ & 172 & 29.9 \\
\hline & $\geq 126 \mathrm{mg} / \mathrm{del}$ & 404 & 70.1 \\
\hline & No & 331 & 81.9 \\
\hline \multirow[t]{2}{*}{ Appropriate meals prepared for DM } & Yes & 431 & 74.8 \\
\hline & No & 145 & 25.2 \\
\hline \multirow[t]{2}{*}{ The difficulty in choosing foods } & Yes & 146 & 25.3 \\
\hline & No & 430 & 74.6 \\
\hline \multirow[t]{2}{*}{ Eating at a restaurant without a good plan in social events } & Yes & 319 & 55.4 \\
\hline & No & 257 & 44.6 \\
\hline \multirow[t]{4}{*}{ Body mass index } & $<18.5 \mathrm{Kg} / \mathrm{m}^{2}$ & I & 0.2 \\
\hline & $18.5-24.99 \mathrm{Kg} / \mathrm{m}^{2}$ & 206 & 35.8 \\
\hline & $25-25.99 \mathrm{Kg} / \mathrm{m}^{2}$ & 299 & 51.9 \\
\hline & $\geq 30 \mathrm{Kg} / \mathrm{m}^{2}$ & 70 & 12.2 \\
\hline
\end{tabular}

Abbreviations: FBG, fasting blood glucose; DM, diabetes mellitus.

affected by the change of community food eating habit and lifestyle and this might be the possible reason for variation in dietary adherence. ${ }^{43}$ The finding was in line with the study conducted in Bahir Dar in which $53.2 \%$ of the participants' adhered to a dietary recommendation. ${ }^{25}$ In multivariable analysis, being college and above educational level was 3.64 times more to adhere to dietary recommendations compared to its counterparts. The possible explanation might be because having a higher education level would probably like with higher information and 
Table 5 Bivariate and Multiple Logistic Regression Analysis of Factors Affecting Dietary Adherence of Type 2 Diabetes Patients Aimed COVID-19 Pandemic ( $\mathrm{N}=576)$

\begin{tabular}{|c|c|c|c|c|c|}
\hline \multirow[t]{2}{*}{ Variables } & \multicolumn{2}{|c|}{ Dietary Adherence } & \multirow[t]{2}{*}{ COR $(95 \% \mathrm{Cl})$} & \multirow[t]{2}{*}{$\operatorname{AOR}(95 \% \mathrm{Cl})$} & \multirow[t]{2}{*}{ P-value } \\
\hline & Yes & No & & & \\
\hline \multicolumn{6}{|l|}{ Sex } \\
\hline Male & 94 & 138 & $1.69(1.21-2.37)$ & $1.26(0.81-1.96)$ & 0.34 \\
\hline Female & 184 & 160 & I & I & \\
\hline \multicolumn{6}{|l|}{ Age in years } \\
\hline$\leq 40$ & 47 & 73 & $1.60(1.06-2.40)$ & $1.35(0.78-2.32)$ & 0.56 \\
\hline$>40$ & 231 & 225 & I & I & \\
\hline \multicolumn{6}{|l|}{ Educational status } \\
\hline Cannot read and write & 177 & 69 & I & 1 & \\
\hline Elementary & 14 & 21 & $3.85(1.85-7.99)$ & $4.47(1.90-10.52)$ & \\
\hline Secondary & 21 & 23 & $2.81(1.46-5.40)$ & $3.50(1.57-7.84)$ & \\
\hline College and above & 66 & 185 & $7.19(4.84-10.68)$ & $3.64(1.59-8.34)$ & $0.00 I^{* *}$ \\
\hline \multicolumn{6}{|l|}{ Residency } \\
\hline Urban & 196 & 262 & $3.05(1.97-4.69)$ & $1.30(1.09-2.42)$ & $0.032 *$ \\
\hline Rural & 82 & 36 & I & I & \\
\hline \multicolumn{6}{|l|}{ Occupation } \\
\hline Gov't employed & 52 & 172 & $5.93(4.06-8.67)$ & $2.38(1.13-4.99)$ & $0.04 *$ \\
\hline Unemployed & 226 & 126 & I & 1 & \\
\hline \multicolumn{6}{|l|}{ Family size } \\
\hline$<5$ & 109 & 188 & $2.65(I .89-3.7 I)$ & I. $27(1.08-1.97)$ & $0.023 *$ \\
\hline$\geq 5$ & 169 & 110 & I & I & \\
\hline \multicolumn{6}{|l|}{ Wealth-status } \\
\hline Low & 46 & $|3|$ & $6.85(4.36-10.78)$ & $3.67(1.54-7.45$ & $0.00 I^{* *}$ \\
\hline Medium & 90 & 108 & $2.89(1.91-4.36)$ & $2.26(1.67-4.54)$ & $0.01 *$ \\
\hline High & 142 & 59 & I & 1 & \\
\hline \multicolumn{6}{|l|}{ Family support } \\
\hline Yes & 187 & 188 & I & 1 & \\
\hline No & 91 & 110 & $1.20(0.85-1.69)$ & $1.09(0.72-1.65)$ & $0.08 \mathrm{I}$ \\
\hline \multicolumn{6}{|l|}{ Family $\mathrm{Hx}$ of DM } \\
\hline No & 212 & 201 & I & 1 & \\
\hline Yes & 66 & 97 & $1.55(1.07-2.24)$ & $2.18(1.14-4.19)$ & $0.032 *$ \\
\hline \multicolumn{6}{|c|}{ The difficulty of choosing food } \\
\hline No & 74 & 72 & I.I4(0.78-1.66) & $1.04(0.62-1.62)$ & 0.073 \\
\hline Yes & 204 & 226 & I & 1 & \\
\hline \multicolumn{6}{|l|}{ Diabetes complication } \\
\hline No & 194 & 201 & I.I2(0.78-I.59) & $1.94(1.04-3.61)$ & $0.003 *$ \\
\hline Yes & 84 & 97 & I & I & \\
\hline \multicolumn{6}{|l|}{ Duration of DM } \\
\hline$<5$ years & 140 & 161 & I & 1 & \\
\hline $5-10$ years & 105 & 97 & $0.80(0.56-1.15)$ & I.I2(0.70-1.77) & 0.056 \\
\hline $10-15$ yeas & 29 & 23 & $0.69(0.38-1.25)$ & $1.20(0.58-2.47)$ & 0.067 \\
\hline$>15$ years & 4 & 17 & $3.70(1.22-11.24)$ & $5.39(1.57-18.52)$ & $0.023 *$ \\
\hline
\end{tabular}

(Continued) 
Table 5 (Continued).

\begin{tabular}{|c|c|c|c|c|c|}
\hline \multirow[t]{2}{*}{ Variables } & \multicolumn{2}{|c|}{ Dietary Adherence } & \multirow[t]{2}{*}{ COR $(95 \% \mathrm{Cl})$} & \multirow[t]{2}{*}{$\operatorname{AOR}(95 \% \mathrm{Cl})$} & \multirow[t]{2}{*}{ P-value } \\
\hline & Yes & No & & & \\
\hline \multicolumn{6}{|l|}{ COVID-19 knowledge } \\
\hline Good & 95 & 166 & $2.42(1.73-3.39)^{*}$ & $4.33(2.7 I-6.92)$ & $0.001 * *$ \\
\hline Poor & 183 & 132 & I & 1 & \\
\hline
\end{tabular}

Notes: Statistically significant $*$ p-value $<0.05$, **p-value $<0.001$.

Abbreviations: COR, crude odds ratio; COVID-19, coronavirus; $\mathrm{Cl}$, confidence interval; AOR, adjusted odds ratio; DM, diabetes mellitus.

awareness about diabetes control as well as COVID-19 pandemic than lower educational level. This finding was supported by the studies conducted in Surate city in which those with higher educational level adhered more than lower-level. ${ }^{21}$ Furthermore, those with higher educational level might have a great chance of reviewing and reading different evidence than lower educational level. For instance, they might read the severity of COVID-19 among diabetes patients found to be 2 to 3 fold increase ${ }^{3}$ and by then increase their dietary adherence to the recommendations. Being a government-employed was found nearly two times more to adhere to a dietary recommendation compared to its counterparts. The possible explanation might be since the vast majority of government employees in our situation can at least read and write the leaflets, banners and some dos and don'ts related to their disease process. This finding was supported by the studies conducted in Yemen. ${ }^{17}$ Being an urban residency was $30 \%$ more to adhere to the dietary recommendation compared to rural. The possible explanation might be because those in Urban have a great chance of information access through different Media. This was supported by the studies conducted in Eastern Ethiopia ${ }^{28}$ and Yemen. ${ }^{17}$ Having a family size of less than five was found to be $27 \%$ more to adhere to the dietary recommendation compared to more than five family sizes. This might be since the lower the family size the lower their expenditure and higher physiologic need satisfaction than larger family size particularly in the era of the COVID-19 pandemic. The locked downtime could probably be challenging with large family size by far. ${ }^{5,41}$ Being in the medium level of wealth-status was nearly two times to adhere to the dietary recommendation compared to its counterparts. This is possibly due to the fact the medium socio-economic status might have great care to their disease proses than high level, put in mined the health care service they might get if contracted by the COVID-19 disease. Having a family history of diabetes was nearly two more to adhere to the dietary recommendation compared to no family history. This might be having a family member with diabetes could be a good source of information about the disease process and how to control blood glucose. The finding was supported by the study conducted in eastern Ethiopia. ${ }^{28}$ Duration of diabetes more than 15 years was found to be nearly five times more to adhere to the dietary recommendation compared to its counterparts. This possibly might be the longer the duration the more the information gained during follow-up. The finding was supported by the studies conducted in Bahir Dar. ${ }^{25}$ Knowledge related to COVID-19 was found to be 4.33 times more to adhere to the dietary recommendation compared to its counterparts. The possible explanation might be those who aware of the COVID-19 impact on diabetes give great self-care that of dietary adherence than a low level of awareness. The finding was supported by the studies. $2,5,41$

\section{Limitations of the Study}

It is an institutional-based cross-sectional study that might not be generalized to the public at large. Moreover, it cannot identify the cause and effect relationships of variables. It did not assess the level of stress and anxiety related to COVID-19 pandemic which could affect dietary adherence.

\section{Conclusion}

Generally, there was considerably limited dietary adherence among the study participants compared to the other studies conducted in and outside of the country. Measures need have to be taken to improve the adherence of dietary recommendations of the participants in the study area. Health professional and other concerned bodies ought to give adequate and clear information regarding the dietary recommendations particularly in this era of COVID-19. There need to strengthen health education about COVID19 and the ways to control sugar level while patients come for service to increase their awareness towards how they 
could control blood sugar level through dietary adherence. Leaflets, posters, and banners need to be done to improve awareness of patients with a full explanation of dos and Don'ts for COVID-19 and diabetes. Awareness creation meetings have to be held by incorporating various target population groups such as patients, families, health bureau officers, health extension workers, and non-governmental organizations. Since diabetic care necessitates collaborative efforts, particularly in the era of COVID-19 pandemic.

\section{Abbreviation}

AOR, adjusted odds ratio; COR, crude odds ratio; COVID-19, coronavirus; DM, diabetes mellitus; IDF, International Diabetes Federation; SPSS, Statistical Package for Social Sciences.

\section{Data Sharing Statement}

For the sake of protecting participants' confidentiality raw data would not be given. However, the summary data are presented in tables and available in the manuscript. The data can be given on reasonable request from the corresponding author.

\section{The Ethical Approval and Consent of Participating}

The study was performed based on the ethical standards of put down the 1964 declaration of Helsinki. The ethical approval was found from the University of Gondar, college of medicine and health sciences, school of nursing research and the ethical review committee. Letter of permission and support was obtained from the University of Gondar Comprehensive Specialized Hospital Clinical Director. The study participants were briefly informed about the purpose, method, advantages expected, and the risk of the study. They had got full information about their rights not to participate or stop to participate in the study at any time. We had got written informed consent from each study participant.

\section{Acknowledgments}

The authors would like to give their deepest appreciations to the University of Gondar, College of Medicine and Health Sciences, School of Nursing research and ethical review committees for the approval of the ethical clearance. The authors also would like to give great thanks to data collectors, supervisors, and study participants.

\section{Author Contributions}

All authors made substantial contributions to conception and design, acquisition of data, or analysis and interpretation of data; took part in drafting the article or revising it critically for important intellectual content; agreed to submit to the current journal; gave final approval of the version to be published; and agree to be accountable for all aspects of the work.

\section{Disclosure}

All the authors declared that there is no competing interest concerning this work.

\section{References}

1. Bello-Chavolla OY, Bahena-López JP, Antonio-Villa NE, et al. Predicting mortality due to SARS-CoV-2: a mechanistic score relating obesity and diabetes to COVID-19 outcomes in Mexico. medRxiv. 2020.

2. Roncon L, Zuin M, Rigatelli G, et al. Diabetic patients with COVID-19 infection are at higher risk of ICU admission and poor short-term outcome. J Clin Virol. 2020;127:104354. doi:10.1016/j. jcv.2020.104354

3. Singh AK, Khunti K. Assessment of risk, severity, mortality, glycemic control and antidiabetic agents in patients with diabetes and COVID-19: a narrative review. Diabetes Res Clin Pract. 2020;165:108266. doi:10.1016/j.diabres.2020.108266

4. Gupta R, Ghosh A, Singh AK, et al. Clinical considerations for patients with diabetes in times of COVID-19 epidemic. Diabetes Metab Syndr. 2020;14(3):211. doi:10.1016/j.dsx.2020.03.002

5. Alromaihi D, Alamuddin N, George S. Sustainable diabetes care services during COVID-19 pandemic. Diabetes Res Clin Pract. 2020;166:108298. doi:10.1016/j.diabres.2020.108298

6. Wang B, Li R, Lu Z, et al. Does comorbidity increase the risk of patients with COVID-19: evidence from meta-analysis. Aging (Albany NY). 2020;12(7):6049. doi:10.18632/aging.103000

7. Guo W, Li M, Dong Y, et al. Diabetes is a risk factor for the progression and prognosis of COVID -19. Diabetes Metab Res Rev. 2020;36(7):e3319. doi:10.1002/dmrr.3319

8. Kumar A, Arora A, Sharma P, et al. Is diabetes mellitus associated with mortality and severity of COVID-19? A meta-analysis. Diabetes Metab Syndr. 2020.

9. Diabetes key facts; 2020. Available from: https://www.who.int/newsroom/fact-sheets/detail/diabetes. Accessed February 23, 2021.

10. Diabetes: lifestyle changes \& prevention; 2020. Available from: https://my.clevelandclinic.org/health/articles/13340-diabetes-lifestyle -changes-prevention. Accessed February 23, 2021.

11. Diabetes in Africa; 2019. Available from: https://www.idf.org/ournetwork/regions-members/africa/diabetes-in-africa.html. Accessed February 23, 2021.

12. Bishu KG, Jenkins C, Yebyo HG, et al. Diabetes in Ethiopia: a systematic review of prevalence, risk factors, complications, and cost. Obes Med. 2019;15:100132. doi:10.1016/j.obmed.201 9.100132

13. Pandey A. Non-adherence to lifestyle (diet and exercise) modification recommendations among the type 2 diabetes mellitus patients in a tertiary level hospital. J Inst Med. 2018;41(2).

14. Salas-Salvadó J, Martinez-González MÁ, Bulló M, et al. The role of diet in the prevention of type 2 diabetes. Nutr Metab Cardiovasc Dis. 2011;21(Suppl 2):B32-48. doi:10.1016/j.numecd.2011.03.009 
15. Forouhi NG, Misra A, Mohan V, et al. Dietary and nutritional approaches for prevention and management of type 2 diabetes. BMJ. 2018;361:k2234. doi:10.1136/bmj.k2234

16. Bazzano LA, Serdula M, Liu S. Prevention of type 2 diabetes by diet and lifestyle modification. J Am Coll Nutr. 2005;24(5):310-319. doi:10.1080/07315724.2005.10719479

17. Alhariri A, Daud F, Saghir SAM. Factors associated with adherence to diet and exercise among type 2 diabetes patients in Yemen. Diabetes Manag. 2017;7(3):264-271.

18. Low adherence to diet and exercise changes in type 2 diabetes; 2013. Available from: https://www.diabetes.co.uk/news/2013/dec/lowadherence-to-diet-and-exercise-changes-in-type-2-diabetes92050840.html. Accessed February 23, 2021.

19. Ganiyu AB, Mabuza LH, Malete NH, et al. Non-adherence to diet and exercise recommendations amongst patients with type 2 diabetes mellitus attending extension II clinic in Botswana. Afr J Prim Health Care Fam Med. 2013;5(1).

20. Pandey A. Non-Adherence to Lifestyle (Diet and Exercise) Modification Recommendations Among the Type 2 Diabetes Mellitus Patients in a Tertiary Level Hospital. 2019.

21. Jadawala HD, Pawar AB, Patel PB, et al. Factors associated with non adherence to diet and physical activity among diabetes patients: a cross sectional study. Natl J Community Med. 2017;8(2):68-73.

22. Akumiah PO, Samuel OA, Azumah Nayembil D, et al. Barriers to adherence to diet and exercise recommendation amongst type 2 diabetes mellitus patients. J Health Med Nurs. 2017;39:48-53.

23. Mumu S, Saleh F, Ara F, et al. Non-adherence to life-style modification and its factors among type 2 diabetic patients. Indian J Public Health. 2014;58(1):40-44. doi:10.4103/0019-557X.128165

24. Muhabuura B. Prevalence and factors associated with non-adherence to diet and exercise lifestyle recommendations among type 2 diabetic patients. Afr J Prim Health Care Fam Med. 2014;4(2):110-120.

25. Tirfie M, Tadesse S, Woldie H, et al. Dietary non-adherence and associated factors among individuals with diabetes who are on treatment follow up at Felege-Hiwot referral hospital, northwest Ethiopia. Heliyon. 2020;6(8):e04544. doi:10.1016/j.heliyon.2020.e04544

26. Ayele AA, Emiru YK, Tiruneh SA, et al. Level of adherence to dietary recommendations and barriers among type 2 diabetic patients: a cross-sectional study in an Ethiopian hospital. Clin Diabetes Endocrinol. 2018;4(1):21. doi:10.1186/s40842-018-0070-7

27. Worku A, Mekonnen Abebe S, Wassie MM. Dietary practice and associated factors among type 2 diabetic patients: a cross sectional hospital based study, Addis Ababa, Ethiopia. SpringerPlus. 2015;4 (1):15. doi:10.1186/s40064-015-0785-1

28. Mohammed AS, Adem F, Tadiwos Y, et al. Level of adherence to the dietary recommendation and glycemic control among patients with type 2 diabetes mellitus in eastern ethiopia: a cross-sectional study. Diabetes Metab Syndr Obes. 2020;13:2605. doi:10.2147/DMSO. S256738

29. Parajuli J, Saleh F, Thapa N, et al. Factors associated with nonadherence to diet and physical activity among Nepalese type 2 diabetes patients; a cross sectional study. BMC Res Notes. 2014;7(1):758. doi:10.1186/1756-0500-7-758
30. Kalyango JN, Owino E, Nambuya AP. Non-adherence to diabetes treatment at Mulago Hospital in Uganda: prevalence and associated factors. Afr Health Sci. 2008;8(2).

31. Teklay G, Hussien J, Tesfaye D. Non-adherence and associated factors among type 2 diabetic patients at Jimma University Specialized Hospital, Southwest Ethiopia. J Med Sci. 2013;13 (7):578-584. doi:10.3923/jms.2013.578.584

32. Zeleke Negera G, Charles Epiphanio D. Prevalence and predictors of nonadherence to diet and physical activity recommendations among type 2 diabetes patients in Southwest Ethiopia: a cross-sectional study. Int J Endocrinol. 2020;2020:1-8. doi:10.1155/2020/1512376

33. Cooper HC, Booth K, Gill G. Patients' perspectives on diabetes health care education. Health Educ Res. 2003;18(2):191-206. doi:10.1093/her/18.2.191

34. Colberg SR, Sigal RJ, Yardley JE, et al. Physical activity/exercise and diabetes: a position statement of the American diabetes association. Diabetes Care. 2016;39(11):2065-2079. doi:10.2337/dc16-1728

35. Angelidi AM, Belanger MJ, Mantzoros CS. Commentary: COVID-19 and diabetes mellitus: what we know, how our patients should be treated now, and what should happen next. Metabolism. 2020;107:154245. doi:10.1016/j.metabol.2020.154245

36. Demasi M. COVID-19 and Metabolic Syndrome: Could Diet Be the Key? Royal Society of Medicine; 2021.

37. Kaliyaperumal K. Guideline for conducting a knowledge, attitude and practice (KAP) study. AECS Illumination. 2004;4(1):7-9.

38. Hackman J, Hruschka D, Vizireanu M. An agricultural wealth index for multidimensional wealth assessments. Popul Dev Rev. 2020. doi:10.1111/padr.12367

39. Abuse, N.I.o.A. and Alcoholism. The Physicians' Guide to Helping Patients with Alcohol Problems. US Department of Health and Human Services, Public Health Service, National; 1995.

40. Demilew YM, Alem AT, Emiru AA. Dietary practice and associated factors among type 2 diabetic patients in Felege Hiwot regional referral hospital, Bahir Dar, Ethiopia. BMC Res Notes. 2018;11 (1):434. doi:10.1186/s13104-018-3531-2

41. Ruiz-Roso MB, Knott-Torcal C, Matilla-Escalante DC, et al. COVID-19 lockdown and changes of the dietary pattern and physical activity habits in a cohort of patients with type 2 diabetes mellitus. Nutrients. 2020;12(8):2327. doi:10.3390/nu12082327

42. Rodríguez-Pérez C, Molina-Montes E, Verardo V, et al. Changes in dietary behaviours during the COVID-19 outbreak confinement in the Spanish COVIDiet study. Nutrients. 2020;12(6):1730. doi:10.3390/ nu12061730

43. Di Renzo L, Gualtieri P, Pivari F, et al. Eating habits and lifestyle changes during COVID-19 lockdown: an Italian survey. J Transl Med. 2020;18(1):1-15. doi:10.1186/s12967-020-02399-5

\section{Publish your work in this journal}

Diabetes, Metabolic Syndrome and Obesity: Targets and Therapy is an international, peer-reviewed open-access journal committed to the rapid publication of the latest laboratory and clinical findings in the fields of diabetes, metabolic syndrome and obesity research. Original research, review, case reports, hypothesis formation, expert opinion and commentaries are all considered for publication. The manuscript management system is completely online and includes a very quick and fair peer-review system, which is all easy to use. Visit http://www.dovepress.com/testimonials.php to read real quotes from published authors. 